\title{
Diacronie
}

Studi di Storia Contemporanea

$N^{\circ}$ 9, 1 | 2012

Quando la classe operaia andava in paradiso

\section{Intervista a Valerio Romitelli}

\section{Luca Bufarale e Fausto Pietrancosta}

\section{Q OpenEdition}

\section{Journals}

\section{Edizione digitale}

URL: http://journals.openedition.org/diacronie/3101

DOI: 10.4000/diacronie.3101

ISSN: 2038-0925

\section{Editore}

Association culturelle Diacronie

\section{Notizia bibliografica digitale}

Luca Bufarale e Fausto Pietrancosta, «Intervista a Valerio Romitelli », Diacronie [Online], № 9, 1 | 2012, documento 17, Messo online il 29 janvier 2012, consultato il 01 mai 2019. URL : http:// journals.openedition.org/diacronie/3101 ; DOI : 10.4000/diacronie.3101 


\title{
Diacronie
}

\section{7/}

\section{Intervista a Valerio Romitelli}

\author{
A cura di Luca BUFARALE, Fausto PIETRANCOSTA \\ Redazione del testo dell'intervista a cura di Fausto PIETRANCOSTA
}

Lo storico Valerio Romitelli ripercorre alcuni momenti fondamentali dei rapporti tra politica, economica, cultura e società nel trentennio 1945-1973. Governance, democrazia progressiva, biopolitica, sono parte dei temi affrontati dallo storico nell'analisi dell'evoluzione delle sinistre europee in una delle fasi cruciali del XX secolo.

\section{Diacronie: Che giudizio dà del periodo definito dalla storiografia francese les trente glorieuses, il trentennio 1945-1973, dal secondo dopoguerra ai prodromi della grande crisi economica degli anni Settanta? Quale analisi si sente di fare sugli aspetti e le caratteristiche che hanno contribuito a definire quegli anni e il rapporto con l'epoca attuale?}

Valerio Romitelli: Questi anni sono per me un riferimento fondamentale. Se oggi c'è ancora un'idea di giustizia sociale, non è perché se ne può leggere in libri di filosofia, di storia o di religione, ma perché c'è ancora memoria di quest'epoca, per certi versi, eguale e contraria all'attuale. Tutto il baccano sollevato dalla letteratura sul "secolo della violenza, delle ideologie e degli orrori"1, o cose simili, ha avuto l'obiettivo tra gli altri di far dimenticare che fino a quarant'anni fa era possibile qualcosa oggi inimmaginabile: l'abbassamento globale delle differenze sociali, ad ogni livello o quasi. Il tutto, certo, sotto l'equilibrio del terrore del ricatto nucleare. Ma oggi le bombe atomiche sono forse scomparse? E di che multipolarismo geopolitico si può mai parlare, quando metà della potenza bellica mondiale sta attualmente in un solo paese, il quale drena anche il quarto della ricchezza dell'intero pianeta ${ }^{2}$ ? Nuovi paesi (Brics $\left.{ }^{3}\right)$ già

\footnotetext{
${ }^{1}$ Cfr. ZACHEO, Egidio, Il secolo della democrazia: politica e diritti nel Novecento, Lecce, Manni editore, 2003; GRILLO, Enzo (a cura di), Karl D. Bracher Il Novecento. Secolo delle ideologie, Bari, Laterza, 2006.

${ }^{2}$ Cfr. POLANSKY, David, L'impero che non c'è. Geopolitica degli Stati Uniti d'America, Milano, Guerini, 2005.

3 Brasile, Federazione russa, India, Cina, Repubblica Sudafricana.
} 
poveri stanno certo divenendo più ricchi dei ricchi, ma anche al loro stesso interno le differenze sociali aumentano e, soprattutto, non offrono idee politiche abbastanza diverse da quelle insegnate dalle suole economiche statunitensi, sempre più o meno in continuità con i tempi di Reagan, malgrado tutti i disastri nel frattempo creati.

Credo sia il caso di riconoscere che le ideologie di tipo novecentesco siano morte e sepolte per sempre. E che questo sia un bene. Ma non va dimenticato che, quando c'erano, le ideologie erano comunque al plurale. Offrivano più visioni del mondo. Mentre oggi impera un unico ed uniforme senso comune: un "pensiero unico", che è riduttivo continuare a chiamare neo-liberale e che merita piuttosto degli aggettivi come info-democratico-cognitivista, come ho ampiamente argomentato in Fuori della società della conoscenza ${ }^{4}$. Tale senso comune si presta alle più svariate versioni, di destra e di sinistra, ma semina comunque e ovunque le sue assurde convinzioni, profondamente ao anti-politiche. Quali, più precisamente?

Lo dico in spericolata, ma necessaria sintesi: anche per merito dell'avvento della comunicazione informatica tra governanti e governati si sarebbe realizzata sempre più democrazia, cosicché ognuno sarebbe potuto e avrebbe dovuto essere manager di se stesso, integrandosi e contribuendo a suo modo alla grande comunità vitale delle relazioni interpersonali; l'unico problema fondamentale sarebbe rimasto quindi quello rappresentato da coloro che, volenti o nolenti, non sono integrabili in questo tipo di comunità interpersonale: o perché non vedono nulla di divino nella "persona" e hanno consuetudini poco comunicative - il fatidico "velo"! - o perché hanno necessità primarie, assolutamente impersonali, come fame, sete e malattie.

Ecco allora che non si trova di meglio che coprirli di filantropia, per quel quasi niente che così si può, oppure, ovunque questa non arriva, perseguitarli o lasciarli morire.

Il guaio è che si tratta di più di un sesto dell'umanità, come, tra gli altri, Paul Collier 5 va denunciando da anni.

\section{D.: Cosa ci può dire in generale circa lo stato delle sinistre europee negli anni cinquanta e sessanta nei diversi contesti nazionali? $\grave{E}$ possibile innanzitutto fare alcune generalizzazioni? Quali sono a suo avviso le differenze più significative tra le sinistre dei vari paesi?}

\footnotetext{
4 ROMITELLI, Valerio, Fuori dalla società della conoscenza. Ricerche di etnografia del pensiero, Roma, Infinito, 2009. 5 COLLIER, Paul, L'ultimo miliardo, Roma-Bari, Laterza, 2009.
} 
V. R.: La divisione più netta è quella imposta dal movimento dei paesi detti non allineati che comincia a crearsi a metà degli anni '50 e che prova a sottrarsi al condomino della Guerra Fredda imposto dalle due superpotenze Usa e Urss ${ }^{6}$. La Cina, ma anche la Jugoslavia e pure l'Egitto di Nasser e l'India di Nehru, ne fanno parte assieme a molti altri. Questo rimescola le carte nelle sinistre di tutto il mondo, in modo differente a seconda dei contesti. Allora quando si parlava di "Terzo mondo" era a questo schieramento politico che si faceva riferimento, mentre il "primo" era quello delle due superpotenze Usa-Urss e il "secondo" quello dei paesi europei e del Giappone. In Italia, le questioni del "terzo mondo" rimasero per molto tempo appannaggio della Chiesa e del suo filantropismo, mentre la sinistra in generale si divise piuttosto tra $\mathrm{i}$ comunisti più vicini a Mosca e i socialisti più propensi a apprezzare le novità "all'americana". Mantenendosi in una visione che io considero ancora ottocentesca del progresso, questi ultimi consideravano la tecnologia la vera protagonista della storia. Ed è da questo filone che prenderà le mosse quella corrente operaista che ha fatto della distinzione fordismo e post-fordismo una chiave di lettura praticamente ontologica della storia. Tutt'oggi non ne mancano certo gli eredi tra gli apologeti del "comune"7.

\section{D.: Da un lato la fase espansiva del capitalismo seguita alla ricostruzione} postbellica, dall'altro i mutamenti sociali e culturali indotti dall'avvento dei fenomeni caratteristici della società di massa. $E$ ancora: le politiche di welfare, la nazionalizzazione dei settori chiave della produzione, la programmazione economica. Come si sono rapportate le sinistre europee ai processi economici in atto nell'arco del trentennio 1945-1973 e come hanno influenzato le dinamiche sociali che ne sono conseguite?

V. R.: Dati i tempi di crisi senza uscita che corrono, credo che occorre essere più che mai netti: smettiamola, una volta per tutte, con la balla, che lo stesso Smith non ha mai veramente sostenuto, secondo la quale i mercati si autoregolano (come tra gli altri ha

\footnotetext{
${ }^{6}$ Cfr. MAURER, Pierre, Le mouvement des pays non-alignés. Essai de sociologie historique, Genève, IUED, 1983; GALEAZZI, Marco, Il PCI e il movimento dei paesi non allineati (19551975), Milano, Franco Angeli, 2011.

7 Cfr. REVELLI, Marco, Oltre il Novecento. La politica, le ideologie e le insidie del lavoro Torino, Einaudi, 2001; HOLDEN, Leonard George, «Fording the Atlantic: Ford and Fordism in Europe», in Business History, 47, 1/2005, pp. 122-127; MAIER, Charles, «Between Taylorism and Technocracy: European Ideologies and the Vision of Industrial Productivity», in Journal of Contemporary History, 5, 2/1970, pp. 27-61; HUGHES, Thomas P., American Genesis: A Century of Invention and Technological Enthusiasm 1870-1970, New York, Viking, 1989.
} 
egregiamente illustrato Giovanni Arrighi in Adam Smith a Pechino ${ }^{8}$ ). Il capitalismo non ha limiti interni. Marx qui continua ad aver ragione. Ingiustizie, guerre e crisi sono le sole cose che ci si deve attendere se non si trova modo di intimorire o distrarre gli imprenditori dalla loro specifica "missione", la quale non può che consistere nel perseguire il massimo profitto. Le religioni hanno svolto a volte la funzione d'orientamento dell'economia, ma è chiaramente la politica cui spetta, secondo la stessa formula secondo la quale l'economia è economia politica. Il problema è che una politica per guidare l'economia non può essa stessa avere come imperativo di essere funzionale all'economia o ancor peggio ad una visione economicista della vita. È questo che avviene con tutte le teorie della governance, comunque declinate. La peculiarità del trentennio 1945-1973 era che il comunismo, sia pur variamente inteso, copriva di rosso mezzo mondo, con le sue ideologie di giustizia sociale più o meno in via di realizzazione. Vere o false che fossero, esse erano abbastanza convincenti da riscuotere grandi consensi anche nell'altra metà del mondo e distrarre i capitalisti dalla loro più intima missione. Prima di farsi concorrenza tra loro e sfruttare la forza lavoro come si deve, cioè al massimo, provvedevano allora a far fronte a quanto temevano di più: il comunismo. Di qui, consumismo, espansione del ceto medio, servizi pubblici "dalla cura alla bara" - come si diceva al tempo di Beveridge ${ }^{9}$-, alti salari, aiuti ai paesi in via di sviluppo e così via, con misure che, con tutte le loro contraddittorietà, in un modo o nell'altro, hanno contribuito a fare di questa straordinaria sequenza un esempio di progressiva eguaglianza sociale: senza precedenti, prima del '45, né prosecuzioni, dopo gli anni '70. Tutte le vicende della sinistre europee in quei trent'anni sono incomprensibili se non si torna a dare il peso che merita il fatto che allora il comunismo era ovunque all'offensiva e in espansione ${ }^{10}$.

\section{D.: In che modo la coscienza antifascista e la consapevolezza delle conquiste democratiche raggiunte hanno condizionato l'azione delle sinistre europee?}

\footnotetext{
8 ARRIGHI, Giovanni, Adam Smith a Pechino. Genealogie del ventunesimo secolo, Milano, Feltrinelli, 2008.

9 William Henry Beveridge, (1879-1963), economista e sociologo redasse nel 1942 un rapporto sulla sicurezza sociale e i servizi alleati (Report of the Inter-Departmental Committee on Social Insurance and Allied Services, meglio conosciuto come "Rapporto Beveridge"), che è servito da base per la riforma del Welfare state britannico messa in atto dal governo laburista dopo le elezioni del 1945.

${ }^{10}$ Cfr. SERVICE, Robert, Compagni. Storia globale del comunismo nel XX secolo, Roma-Bari, Laterza, 2008 (ed. orig. Comrades. A world history of Communism, London, MacMillan, 2007).
} 
V. R.: Su questi temi so di andare molto controcorrente. Tengo particolarmente al fatto che la storia abbia l'obbligo di occuparsi anzitutto di singolarità storiche, e quindi limitare il più possibile le generalizzazioni: il fare di un caso il modello per la comoda spiegazione di infiniti altri. Simili banalizzazioni purtroppo fanno parte della stessa cosiddetta professionalità degli storici, e ciò avviene più che mai a proposito di fascismo e antifascismo. Per far di meglio il fascismo propriamente detto non può che essere considerato una sequenza della storia d'Italia che dura vent'anni e che tra l'altro si apre e si chiude da sola ${ }^{11}$. Cosi, pure l'esistenza politicamente efficace dell'antifascismo va ristretta a quei pochi anni durante i quali davvero è stato operativo: tra il '42, quando i comunisti sono costretti a vedere vanificata l'alleanza coi nazisti (sancita nel '39) e allora si uniscono con quegli anglo-americani coi quali romperanno nel '47, all'innesco della Guerra Fredda. In seguito, sono convinto che l'antifascismo sia contato infinitamente meno dell'opposizione tra comunismo e anticomunismo, anche a sinistra. E prima, a fascismo esistente, occorre ammettere che di antifascismo politicamente efficace ce n'è stato praticamente solo all'estero (in Francia e in Spagna, soprattutto). Il fatto cruciale è che in Italia la coscienza antifascista e democratica ha ampiamente sottovalutato il fatto che mentre il fascismo si era fatto e disfatto da solo, la Repubblica italiana è nata sotto quei pesantissimi condizionamenti internazionali che hanno gravato su tutta la sua storia, punteggiandola di nefasti eventi misteriosi e limitandone non poco la sovranità. Scusandomi per la seconda autocitazione: di tutto ciò ho scritto nel volume collettaneo La democrazia in Italia ${ }^{12}$.

D.: L'integrazione europea e la tensione tra i blocchi, sono stati due nodi che hanno costituito un'occasione di confronto e di scontro tra le varie anime della sinistra. Quali punti di contatto o di differenziazione hanno prodotto il processo di costruzione della Comunità Europea e la contestuale rilettura della recente storia del continente sui partiti, sulle classi dirigenti e sull'opinione pubblica di sinistra in Europa?

V. R.: Per così dire, la sfortuna dell'Ue è stata di essere possibile solo col crollo del muro di Berlino. Dunque di venire costruita nell'euforia dell'anticomunismo trionfante,

${ }^{11}$ Cfr. DE FELICE; Renzo, Le interpretazioni del fascismo, Bari, Laterza, 1969; GENTILE, Emilio, Fascismo. Storia e interpretazione, Bari, Laterza, 2002; TARCHI, Marco, Il fascismo. Teorie, interpretazioni, modelli, Bari, Laterza, 2003; NOLTE, Ernst, I tre volti del fascismo, Milano, Mondadori, 1974.

12 ROMITELLI, Valerio, La democrazia in Italia, Napoli, Cronopio, 2010. 
quando l'eco degli incredibili successi del monetarismo reaganiano era ancora al massimo. Perché bisogna ben ricordarselo che si tratta anzitutto di una Unione essenzialmente Monetaria: Ume! In un'altra epoca, chi mai se la sarebbe potuta immaginare un'alleanza così ambiziosa - strategicamente, istituzionalmente, socialmente - tra Stati così importanti e così diversi, la quale pretendesse di fondarsi quasi esclusivamente su banche e denaro, oltre che su misure restrittive per l'immigrazione? La Germania dell'Ovest, come insegna magistralmente Foucault - nel ben noto testo del '79, La nascita della bio-politica ${ }^{13}$ - era stata sì, nel secondo dopoguerra, il laboratorio di una ricostruzione concepita e attuata in termini principalmente economici e a-politici, ma ciò era avvenuto per due condizioni particolari ben evidenti: di non avere alcun riferimento politico nazionale disponibile, perché tutti sfregiati dall'infamia nazista; di avvenire sotto il controllo militare degli anglo-americani e direttamente alla frontiera dell'impero sovietico. Ricostruendosi come unione essenzialmente monetaria, economica, l'Ue negli anni '9o ha ripreso un simile cammino, quasi come se fosse commissariata da quegli stessi Usa che negli anni '8o erano stati protagonisti nel portare al disfacimento l'Impero sovietico (allora detto fantascientificamente "del male”). Del resto l'attuale unione monetaria dell'Europa ha come protagonista quella stessa Germania che ai tempi di Hitler l'aveva già unificata, per altro senza incontrare resistenze insormontabili. Le grandi conquiste che si attribuiscono al modello socialdemocratico europeo, non per puro caso avvengono prima che l'Ue esista ${ }^{14}$. Troppo spesso si sottovaluta quanto tali conquiste siano state condizionate dalla pressione allora svolta dall'esistenza del comunismo in mezzo mondo e quindi degli entusiasmi e delle paure che esso suscitava nell'altra metà. E non è sempre per puro caso che al venire meno di tale pressione la sinistra di tradizione socialdemocratica in molti casi si sia dimostrata propensa a far sue le ricette

13 «Con tale termine si indica un concetto usato per la prima volta da Georges Bataille all'inizio del Novecento, divenuto centrale nel dibattito filosofico in seguito all'uso che ne ha fatto Michel Foucault a partire da metà anni settanta. Nella concezione di Foucault la biopolitica è il terreno in cui agiscono le pratiche con le quali la rete di poteri gestisce le discipline del corpo e le regolazioni delle popolazioni. È un'area d'incontro tra potere e sfera della vita. Un incontro che si realizza pienamente in un'epoca precisa: quella dell'esplosione del capitalismo». Emblematica a riguardo l'espressione «Si potrebbe dire che al vecchio diritto di far morire o di lasciar vivere si è sostituito un potere di far vivere o di respingere nella morte» (FOUCAULT, Michel, La volontà di sapere, Milano, Feltrinelli, 1978). Cfr. MARZOCCA, Ottavio (a cura di), Biopolitica e liberalismo: detti e scritti su potere ed etica, 1975-1984, Milano, Medusa, 2001.

${ }_{14}$ Cfr. OLIVI, Bino, L’Europa difficile. Storia politica dell'integrazione europea, Bologna, Il Mulino, 2001. 
neoliberali. Dire che l'Ue va oggi difesa per il modello socialdemocratico che le sarebbe proprio è parecchio equivoco ${ }^{15}$.

D.: Quale influenza hanno avuto i movimenti anticolonialisti e le rivoluzioni in Asia, Africa e America Latina (rivoluzione cinese, rivoluzione cubana, guerra del Vietnam, lotte di liberazione nelle colonie portoghesi etc.) nel dibattito all'interno delle sinistre europee e nella ridefinizione del concetto di imperialismo? A suo avviso la seconda metà degli anni settanta, con la crisi dei movimenti di liberazione e la loro degenerazione in regimi burocratico-autoritari, ha costituito una frattura importante nella visione che le sinistre europee avevano del "Terzo Mondo" e delle sue possibilità di emancipazione?

V. R.: Il crollo del comunismo sovietico e lo svuotamento di quello cinese ${ }^{16}$, hanno lasciato il mondo intero orfano di un'idea politica realmente sperimentabile di giustizia sociale. Ci si lamenta del fatto che la globalizzazione dei mercati non sia stata accompagnata da politiche in grado di dirigerli. Ma la possibilità di tali politiche non si creerà mai semplicemente facendo opposizione, reagendo a quelle inconfessabili e nascoste dietro la favola dell'autoregolazione del capitalismo. Se si pensa che ci voglia più giustizia sociale in un mondo in cui ce ne è sempre meno si tratta di cambiare terreno rispetto a quello puramente economico più o meno democraticamente rappresentato. Dei cantieri ove si tenta di elaborare l'idea che "un altro mondo è possibile" continuano ad aprirsi e chiudersi, soprattutto nelle periferie del mondo. Nord Africa e Medio Oriente ne stanno dando parecchi esempi. Ma i sostegni che vengono dalle più "grandi democrazie", come l'Italia, non sono che ingerenze, intrighi e guerre "umanitarie", come in Libia. Guerra, quest'ultima, che in Italia è stata caldeggiata soprattutto dalla Sinistra. Per di più in sfregio ad accordi appena presi in

15 MASULLI, Ignazio, Welfare State e patto sociale in Europa. Gran Bretagna, Germania, Francia, Italia 1945-1985, Bologna, CLUEB, 2003. Cfr. anche BORIONI, Paolo, «La socialdemocrazia nordica e la "sfida democratica al capitalismo"», in Diacronie. Studi di Storia Contemporanea : Quando la classe operaia andava in paradiso, $\mathrm{N}^{\circ} 9,1 \mid 2012$, URL: < http://www.studistorici.com/2012/o2/13/borioni_numero_9/ >.

${ }_{16}$ Cfr. GRAZIOSI, Andrea, L'Unione Sovietica 1914-1991, Bologna, Il Mulino, 2011; TOMBA, Luigi, Storia della repubblica popolare cinese, Milano, Mondadori, 2002. 
cui l'Italia si impegnava con questo paese a non ripetere più aggressioni come quelle, infami, avvenute giusto un secolo fa ${ }^{17}$.

\section{D.: In che modo i partiti della sinistra si sono rapportati ai movimenti collettivi della fine degli anni sessanta? Quali relazioni virtuose, criticità o distanze sono nate e si sono consolidate tra la sinistra "istituzionale" e questi movimenti?}

Per attenerci alla seconda metà degli anni '70 in Italia credo che le cose siano andate nel peggiore dei modi. L'ipotesi "eurocomunista" che passava per il "Compromesso storico" era sicuramente un tentativo di fare dell'Italia il centro di rinnovamento dello stesso comunismo, di fronte alla degenerazione sovietica e alla controrivoluzione cinese. Ma il prezzo fu proprio di sacrificare i movimenti che allora ancora tentavano delle loro sperimentazioni, sia pur confuse, ma più vicine al sociale, sempre più o meno in nome dello stesso comunismo. Anche l'insorgere del femminismo o di altre nuove rivendicazioni per me è infatti impensabile senza l'onda lunga dei movimenti più politici. In mezzo c'è stato, certo, il trauma profondissimo e sempre poco chiarito dell'omicidio Moro. Ma il modo in cui ha reagito lo Stato, soprattutto per iniziativa del Pci, ha finito per sterilizzare l'Italia da qualsiasi iniziativa politica extraparlamentare. Quel che si chiama la società civile ne è uscita irrimediabilmente impoverita. Lo si vede ancora oggi che è praticamente impossibile parlare di '68, di '77 e di tutti quei movimenti che vi fanno ancora riferimento, senza parlare di terrorismo, mentre credo siano cose da tenere sempre ben distinte ${ }^{18}$.

\section{D.: Come la crisi economica degli anni Settanta ha contribuito a ridefinire il panorama politico delle sinistre in Europa?}

V. R.: Sicuramente si è trattato di una svolta epocale importante, perché fino ad allora l'orizzonte "di sinistra" era quello di estendere le conquiste di giustizia sociale ottenute soprattutto a partire dal '45, mentre con la crisi venne il tempo della ritirata e della

${ }_{17}$ Cfr. DEL BOCA, Angelo, Gli italiani in Libia, Roma-Bari, Laterza, 1986; DEGL'INNOCENTI, Maurizio, Il socialismo italiano e la guerra di Libia, Roma, Editori Riuniti, 1976; GRAMELLINI, Fabio, Storia della Guerra Italo-Turca 1911-1912, Forlì, Acquacalda Comunicazioni Srl, 2005.

18 Cfr. GIACHETTI, Diego, I partiti della nuova sinistra: origini, sviluppo, epilogo, in BILLI, Fabrizio (a cura di), Gli anni della rivolta. Prima, durante e dopo il '68, Milano, Punto Rosso, 2001, pp. 85-102. 
difensiva. È in questo momento che non solo il paradigma della rivoluzione, ma anche quello delle "grandi riforme di struttura" mostrano tutta la loro obsolescenza. Così un lunghissimo ciclo di sperimentazioni politiche si conclude. I regimi comunisti allora sono ancora in piedi, ma non entusiasmano più, così come fanno sempre meno paura. È da allora che sono cominciate a comparire le ipotesi che una politica di maggior giustizia sociale la si può fare solo "nel basso", unendo e organizzando chi ha meno potere, per non disperdersi o piegarsi del tutto di fronte a chi ne ha enormemente di più. A proposito dell'emergere di queste ipotesi è esemplare la raccolta di testi di Foucault - che per altro non mi ha mai convinto del tutto - Bisogna difendere la società $^{19}$.

\section{D.: Si può ritenere corretta una lettura che vede l'uso del termine "sinistra"} non solo nell'accezione partitica ma soprattutto come espressione dell'incontro tra culture politiche, orientamenti di pensiero e visioni del mondo, spesso anche molto differenti tra loro? Che ruolo ebbero in tal senso le realtà non propriamente partitiche nella ridefinizione della sinistra?

V. R.:Per me si tratta di un termine grossolano, a precipuo uso elettorale, cui personalmente ricorro molto raramente. Esso amalgama realtà collettive così diverse, che a volte si sono combattute persino più aspramente di quanto non abbiano fatto con la destra. È avvenuto, ad esempio, nella guerra civile spagnola ${ }^{20}$, tra i partigiani italiani e francesi o anche attorno al '68 e al '77. Per di più, richiamarsi alla sinistra in genere oggi può essere quanto mai equivoco. Ad esempio, quando in suo nome si tollera l'omologazione del comunismo al nazismo sotto la categoria di totalitarismo. Una categoria, quest'ultima, che deve molto del suo successo al famoso libro della Arendt. Il quale è certo molto interessante, ma è un libro da Guerra fredda ${ }^{21}$.

19 BERTANI, Mauro, FONTANA, Alessandro (a cura di), Bisogna difendere la società, Milano, Feltrinelli, 1998, pp. 36 et seq.

${ }^{20}$ Cfr. sulla Guerra civile spagnola si veda il numero monografico di «Diacronie»: Diacronie. Studi di Storia Contemporanea : «Spagna Anno Zero» La guerra come soluzione, $\mathrm{N}^{\circ} 7,3 \mid 2011$, URL: < http://www.studistorici.com/dossier/n-7-luglio-2011/ >.

${ }^{21}$ ARENDT, Hannah, Le origini del totalitarismo, Milano, Edizioni di Comunità, $1967 .{ }^{22}$ Cfr. DI LORETO, Pietro, Togliatti e la doppiezza: il PCI tra democrazia e insurrezione, 1944-49, Bologna, Il Mulino, 1991; ID., Alle origini della crisi del PCI: Togliatti e il legame di ferro, Roma, EUROMA, 1988; LEHNER, Giancarlo, Palmiro Togliatti Biografia di un vero stalinista, Milano, SugarCo, 1991; AGA ROSSI, Elena, ZASLAVSKY, Victor, Togliatti e Stalin. Il PCI e la politica estera staliniana negli archivi di Mosca, Bologna, Il Mulino, 1997. Per una prospettiva 
Riguardo a Stalin, senza temere lo scandalo, mi confronto piuttosto con Althusser, che è stato e resta un mio maestro, con Losurdo, Canfora, Groys, ma anche con le note critiche che a suo tempo gli rivolgeva Mao... Questo solo per fare qualche nome tra coloro che hanno capito come una simile figura, sicuramente sinistra, abbia avuto comunque un ruolo non sottovalutabile - non solo esecrabile e puramente distruttivo come Hitler - nella storia del secolo scorso.

La stessa svolta di Salerno, la "democrazia progressiva", il "partito di tipo nuovo" che passano come invenzioni di Togliatti e che stanno all'origine di tutta la storia della sinistra comunista italiana dal ' 44 in poi, sono state fuori di ogni dubbio quantomeno concordate direttamente con lo stesso Stalin. Nel bene e nel male. Non ne sono infatti per nulla un sostenitore. Ma senza capire quel che diventa la strategia sovietica fin dal finire degli anni '3o sono convinto che non si possa capire molto neanche della storia dell'Italia repubblicana ${ }^{22}$.

Non vogliatemente per una terza autocitazione, ma di tutto questo mi sono dilungato in Per un altro bilancio del cosiddetto secolo della violenza nel quaderno Sulla violenza ${ }^{23}$.

\section{D.: Quale ruolo hanno svolto le diverse generazioni politiche nella costruzione dell'identità della sinistra?}

V. R.: Conoscete il libro dell'antropologo Remotti, Contro l'identità? Lo condivido in pieno. Identità, aristotelicamente, vuol dire "non contraddizione"24. Quando mai parlando di soggettività, di qualunque di tipo di soggettività, ma soprattutto di quella politica, si può parlare di qualcosa di fisso, stabile, sempre uguale a se stesso nel mutare del tempo? Forse se ne può parlare nel caso dei dogmatismi ideologici, che credono di andare sempre dritti per la loro strada, cosicché più il tempo passa, più si ritrovano a fare cose del tutto diverse dalle loro intenzioni. Insomma, io evito sempre accuratamente di usare questo termine, "identità". Semmai utilizzo "identificazione",

diversa, che sottolinea maggiormente i motivi di autonomia nelle scelte di Togliatti in quel periodo, si veda AGOSTI, Aldo, Togliatti. Un uomo di frontiera, Torino, UTET, 2003.

${ }_{22}$ Cfr. DI LORETO, Pietro, Togliatti e la doppiezza: il PCI tra democrazia e insurrezione, 194449, Bologna, Il Mulino, 1991; ID., Alle origini della crisi del PCI: Togliatti e il legame di ferro, Roma, EUROMA, 1988; LEHNER, Giancarlo, Palmiro Togliatti Biografia di un vero stalinista, Milano, SugarCo, 1991; AGA ROSSI, Elena, ZASLAVSKY, Victor, Togliatti e Stalin. Il PCI e la politica estera staliniana negli archivi di Mosca, Bologna, Il Mulino, 1997. Per una prospettiva diversa, che sottolinea maggiormente i motivi di autonomia nelle scelte di Togliatti in quel periodo, si veda AGOSTI, Aldo, Togliatti. Un uomo di frontiera, Torino, UTET, 2003.

23 ROMITELLI, Valerio, «Per un altro bilancio del cosiddetto secolo della violenza», in BROOKS, Peter et al., Sulla violenza, Napoli, Cronopio, 2009, pp. 51-79.

24 REMOTTI, Francesco, Contro l'identità, Roma-Bari, Laterza, 2001. 
ad indicare un processo soggettivo che al tempo stesso implica una qualche disidentificazione. Ciò in cui la sinistra comunista e socialista si è identificata è stata la favola togliattiana che la dipingeva in continuità con la tradizione nazional-popolare dell'anteguerra, dimenticando la svolta, quella iniziata a Salerno nell'aprile del '44, che l'aveva rifondata radicalmente.

La mia generazione, quella che aveva più o meno vent'anni attorno al fatidico '68 ha vissuto un cambiamento epocale straordinario ${ }^{25}$. Esso non ha riguardato solo il passaggio dal mondo dei "trent'anni gloriosi" al mondo del neoliberismo selvaggio, ma ha riguardato soprattutto il modo di porsi nei confronti della politica: se fino alla fine gli anni '70 era un modo appassionato, che stimolava, specie tra i giovani, grande impegno di studio e di svariate attività, soprattutto "nel basso" della società, accanto ad operai, poveri ed emarginati, senza ambizioni di carriera o candidature personali, tutto in seguito e repentinamente è cambiato. Dopo la devastante parentesi terroristica, a partire dagli anni '8o è iniziata l'epoca del disimpegno approdato ai nostri giorni all'apatia e all' antipatia nei confronti della politica. Se oggi c'è impegno, infatti, si vuole sempre sociale: magari anche di conflittualità, di rivolta e di rivendicazione, ma sempre sociale. E se c'è interesse per la politica è solo interesse per la caccia di consensi e voti a fini elettorali.

Una delle colpe politiche maggiori della mia generazione, trovo stia nel non ricordare a sufficienza il tempo in cui la politica era un affare che poteva appassionare chiunque, intellettualmente e praticamente, ma soprattutto a distanza da ogni potere. Anziché ripensare come oggi possa essere rinnovabile simile tipo di impegno, si preferisce archiviare ' 68 e dintorni come una stagione di belle utopie giovanili in gran parte annichilite appena confrontatesi con la dura realtà.

D.: Socialdemocratici, laburisti e socialisti, comunisti ortodossi e dissidenti, anarchici e trotskisti: è condivisibile la lettura in base alla quale tra queste anime e questi gruppi della sinistra contaminazioni e osmosi furono più frequenti di quanto normalmente si ritenga? La classica distinzione tra "riformisti" e "rivoluzionari" può sempre essere adottata in maniera univoca e monolitica o vanno proposte distinzioni che configurino

${ }^{25}$ Sul Sessantotto si vedano anche DE BERNARDI, Alberto, FLORES, Marcello, Il Sessantotto, Bologna, Il Mulino, 1998; MARINO, Giuseppe Carlo, Biografia del Sessantotto, Milano, Bompiani, 2004; TOLOMELLI, Marica, Il Sessantotto. Una breve storia, Roma, Carocci, 2008. 


\section{diversi e a volta paralleli percorsi delle varie realtà della sinistra in Europa in base al paese e al momento storico?}

V. R.: Personalmente sto tentando da anni di reimpostare la storia della politica, più in particolare novecentesca, non in termini di interessi di classe in lotta tra loro, delle loro rappresentanze elettorali, ma neanche in termini di interessi individuali o di lobby in concorrenza tra loro. Al centro cerco di porre la questione delle passioni, non come pura irrazionalità, ma come insieme contingente di logiche che non si lasciano mai sintetizzare in un'unica logica storico-conflittuale o sistemica. In tale ricerca mi giovo di svariate ispirazioni, tratte non solo dal marxismo, ma anche dalla psicanalisi lacaniana, dalla filosofia di Badiou, dall'antropologia di Lazarus, solo per fare qualche nome, cui va comunque aggiunto quello Hirschman e di quel suo straordinario libro che si intitola appunto Le passioni e gli interessi ${ }^{26}$, decisivo per una rilettura di tutto il pensiero politico moderno prima della fine del Settecento. L'idea di fondo che ne ricavo è che senza l'amore e l'entusiasmo di vaste popolazioni per un'idea di giustizia sociale come il comunismo e il socialismo nessuna politica in loro nome sarebbe mai stata possibile. Dunque, in una simile prospettiva, è il segreto di questi sentimenti unificanti, prima che bellicosi, a dovere essere indagato. Ecco allora che la costruzione del socialismo in Urss, come poi quella cinese, non possono essere sottovalutate, anche per tutte le mitologie d'amore e odio che, a torto o a ragione, ne sono scaturite. Senza di esse sono convinto che il marxismo sarebbe rimasto come appunto l'anarchismo una corrente di pensiero sempre rispettabile, ma inevitabilmente ottocentesca, senza alcun impatto decisivo sui destini del Novecento. Tutte le dissidenze, come il trotskismo, che sono state sacrificate dalle principali sperimentazioni di comunismo e socialismo non perdono così importanza, ma il loro sacrificio è appunto da vedere come prezzo, a volte esorbitante e controproducente, di tali sperimentazioni, nonché delle loro immense ripercussioni ${ }^{27}$.

Quanto alla controversa faccenda tra "riforme" e "rivoluzione" un punto da chiarire credo stia proprio nella storia dell'Urss. Solo qualche battuta in merito. Per non restare in guerra col resto del mondo, negli anni Trenta, anche a seguito della crisi del '29, gli esperti sovietici teorizzarono, sia pur in modo tormentato, l'imminente "crollo del capitalismo", che divenne anche la dottrina dell'Internazionale. Da quel momento in poi si ritenne che la via maestra della storia, il suo corso normale, avrebbe portato al

${ }^{26}$ HIRSCHMAN, Albert Otto, Le passioni e gli interessi. Argomenti politici in favore del capitalismo prima del suo trionfo, Milano, Feltrinelli, 1979.

27 Per un quadro complessivo dei comunismi "dissidenti" cfr. POGGIO, Pier Paolo (a cura di), L'altronovecento. Comunismo eretico e pensiero critico, Milano, Jaca Book, 2010. 
comunismo. Cosicché ci si poteva anche accordare con le maggiori potenze capitalistiche ritenute tutte agonizzanti, per attenuarne gli eventuali colpi di coda, mentre i veri nemici detestabili divennero gli "estremisti", le "spie", i "sabotatori", i "traditori”, con tutte le note conseguenze devastanti. La ricostruzione post bellica, con l'immenso prestigio guadagnato a Stalingrado, con la conquista di tutta l'Europa dell'est e poi con la nascita del socialismo cinese nel '48, venne inizialmente vista come una possibile ricostruzione globalmente socialista. Essa, per bocca dello stesso Stalin, sarebbe potuta avvenire addirittura anche sotto la corona d'Inghilterra, compatibilmente con essa. L'eventualità di qualsiasi rivoluzione era già quindi del tutto esclusa. Più che mai lo fu con l'innesco della Guerra fredda. In simili condizioni qualsiasi rivoluzione rilevante avrebbe infatti comportato, né più, né meno, il rischio di una terza guerra mondiale.

Il tutto per dire che se tra i comunisti come quelli italiani restava l'amore per la rivoluzione si trattava per lo più dell'amore per un mito, che lo stesso partito non infrangeva del tutto per calcoli di consenso elettorale.

Attorno al '68 la ripresa alla grande del tema rivoluzionario è completamente un'altra storia: decisamente ideologica, di rottura interna con l'ortodossia comunista, come lo fu a quel tempo la rivoluzione culturale cinese lanciata esattamente contro la burocrazia del partito, con tutti gli echi mondiali che ciò suscitò.

Da ultimo, vorrei menzionare una questione assai complessa riguardo alla stessa parola "rivoluzione". Parlandone nel 1792 Robespierre accosta quella "politica e morale" 28 , che dice ancora da compiere, a quella astrofisica, "copernicana". Così viene indicato qualcosa di molto profondo: che l'orizzonte della politica doveva cominciare ad essere quello dell'“universo infinito" scoperto dalla fisica astronomica, e non quello del "mondo chiuso" della cosmologia tolemaica - per usare i due termini illuminanti che titolano il formidabile libro di Alexander Koyrée ${ }^{2}$. Il che è esattamente il contrario di quello che sostiene, ad esempio, Carl Schmitt, assieme al suo foltissimo seguito, specie nella sinistra italiana, quando sostiene cose come che «tutti i concetti politici sono concetti teologici secolarizzati»30. "Rivoluzione" è dunque una o la parola che meglio

${ }^{28} \mathrm{Si}$ veda ROBESPIERRE, Maximilien, Pour le bonheur et pour la liberté: discours, Paris, La Fabrique, 2000. Cfr. RUDÉ, George, Robespierre, Portrait of a Revolutionary Democrat, London, Collins, 1975; SCURR, Ruth, Fatal Purity. Robespierre and the French Revolution, London, Chatto \& Windus, 2006; HAMEL, Ernest, Histoire de Robespierre, Paris, Lacroix, 1865; BELLOC, Hilaire, Robespierre (1758-1794), London, Nisbet, 1901; MATHIEZ, Albert, Etudes sur Robespierre (1758-1794), Paris, Editions Sociales, 1958.

29 KOYRÉ, Alexandre, Dal mondo chiuso all'universo infinito, Milano, Feltrinelli, 1970.

${ }_{30}$ Cfr. SCHMITT, Carl, Teologia politica, Vol. 2, La leggenda della liquidazione di ogni teologia politica, Milano, Giuffré, 1992; CAVALLO, Riccardo, Le categorie politiche del diritto. Carl Schmitt e le aporie del moderno, Acireale-Roma, Bonanno, 2007; GALLI, Carlo, Genealogia 
attesta la necessità mai veramente ottemperata di una modernizzazione della politica, del suo pensiero, delle sue condizioni sperimentali. Ora a me pare che questa stessa parola oggi abbia perso del tutto senso, al punto che può essere "colorata" e “infiorettata" per venire strumentalizzata per i peggiori intrighi internazionali. Ma non ha perso d'attualità il senso della sua prescrizione di dovere ancora modernizzare un pensiero politico che invece ragiona sempre per lo più come se si trovasse ancora in un "mondo chiuso".

D.: Dal suo punto di vista, quali sono gli errori che la sinistra italiana ha compiuto nel trentennio $1945-1973$ ? Quali sono stati i nodi non risolti che poi l'hanno portata alla crisi della fine degli anni ottanta? Quali meriti e quale ruolo positivo hanno giocato le sinistre nel processo di ricostruzione materiale e morale in Italia dopo il ventennio fascista e la guerra?

V. R.: Più che di errori, parlerei piuttosto di esaurimento in quegli anni del partito come corpo in cui si organizzano le passioni politiche. Esaurimento che ha riguardato tutti i partiti, non solo quelli di sinistra. Con tale conclusione sono scomparse anche le ideologie che tenevano i partiti in contatto col sociale, con le diverse parti o classi del sociale. Così, oggi quel che pensa la gente che non conta, quella che più fatica e soffre, $\mathrm{e}$ che è sempre la stragrande maggioranza, è divenuto sempre più misterioso. A ciò si cerca di ovviare con la caccia di informazioni su chiunque, tramite sondaggi, indicizzazioni del "gradimento" e così via. Ma questo modo di trattare la gente come numero serve solo a mercati ed elezioni, mentre si aggrava sempre più la distanza tra $\mathrm{i}$ veri centri di potere e l'immensa maggioranza delle popolazioni. Si comprende così come tra di esse, in mancanza d'altro, siano ripresi alla grande miti religiosi, identitari e xenofobi.

D.: Quale ruolo ha giocato nella definizione di un'identità comune della sinistra europea la peculiarità della sinistra italiana, della sua storia così diversa da quella delle tradizioni europee - come quella socialista francese, quella socialdemocratica tedesca e scandinava, quella laburista inglese?

della politica. Carl Schmitt e la crisi del pensiero politico moderno, Bologna, Il Mulino, 1996; NICOLETTI, Michele, Trascendenza e potere. La teologia politica di Carl Schmitt, Brescia, Morcelliana, 2002. 
V. R.: Oggi gli stessi leader massimi dell'Ume sostengono che va rifondata radicalmente, ma non si vede come ciò possa avvenire se non si trovano idee, corpi organizzati e pratiche politiche alternative a quelle molto aggressive nascoste dietro le valutazioni finanziarie delle famigerate agenzie statunitensi che si sono arrogate il privilegio di elaborarle. Meno che mai credo possa dare contributi positivi quella sinistra italiana, che in cinquant'anni è spensieratamente passata dal pro al contro e viceversa, in merito a questioni cruciali come comunismo, atlantismo e neoliberalismo, girando quasi sempre nel senso del vento più forte e tenendosi sempre più aggrappata ad un "centro" inesistente.

\section{D.: Lei è un attento osservatore della realtà politica dell'Italia: come vede l'ormai più che decennale crisi d'identità della sinistra italiana e la difficile unità delle sue molte anime?}

V. R.: Il problema resta, ci vogliono nuove idee di giustizia sociale e nuovi corpi organizzati in grado di sperimentarle. Tutto ciò non può che avvenire non tanto "dal basso", ma "nel basso", tra le popolazioni che più faticano e soffrono e col concorso di elaborazioni intellettuali che sappiano produrre vere conoscenze politiche. Così, in effetti, da niente, da zero in termini di potere, si è formato a suo tempo quel socialismo che il marxismo ha voluto scientifico e che ha inciso sulla storia dei secoli scorsi infinitamente molto più di quanto si ammette oggi. Tale cammino non credo sia oggi ripercorribile o rifondabile, ma di sicuro c'è da trarne sempre molti insegnamenti.

A questo proposito penso, anche alle Ong alle quali i governi hanno delegato la soluzione dei maggiori problemi sociali, specie laddove povertà e penurie varie non cessano di aumentare. Tali Organizzazioni spesso sono "non governative" solo sulla carta e soprattutto hanno l'obbligo, non solo di non avere scopi di lucro, ma anche di non avere scopi politici propri. Ecco, tanto per fare un esempio, qualcosa in cui sperare potrebbe essere proprio una sempre maggiore e più qualificata politicizzazione delle Ong più degne di questo nome e a partire dalle loro esperienze più profonde.

D'altra parte, solo per dare un esempio di come credo sia sempre possibile un "ottimismo della volontà" faccio presente un'attività di ricerca del tutto minimale in cui sono impegnato da più di dieci anni, in quanto docente e ricercatore anche di Metodologia delle scienze sociali. Si tratta delle inchieste condotte dal Grep (Gruppo di ricerca di etnologia del pensiero) sulle parole e il pensiero delle popolazioni che faticano a decidere della loro esistenza. Tali ricerche sono sempre condotte tramite 
interviste molto approfondite e all'interno di luoghi di lavoro o di fruizione di servizi sociali fondamentali. L'ipotesi di fondo è che la fatica di tali popolazioni renda possibile parole e pensieri altrimenti impossibili e del tutto sconosciute sia ai governanti, sia agli esperti di questi stessi luoghi. Presentando rapporti d'inchiesta il Grep ${ }^{31}$ è convinto di potere offrire a chi ne ha il potere l'opportunità di migliorare in senso davvero democratico quelle politiche sociali che diversamente non trovano razionalità, se non nei calcoli economici o, nel migliore dei casi, in volontà filantropica miope o simulata. Fermo restando, ovviamente, che simili attività d'inchiesta si limitano a proporre un sapere che resta impotente se non c'è una politica che lo fa suo e lo fa valere presso i governanti. Anche da questo punto di vista resta quindi sempre aperto il problema di come sia possibile una politica interna alle pieghe più oscure del sociale. Non credo affatto, in ogni caso, che la soluzione stia nelle elezioni, né nei loro preparativi, né nelle governance risultanti.

\section{D.: E in chiave europea di quale identità possiamo parlare oggi per la sinistra? Il riformismo è l'unico destino possibile per la sinistra europea?}

V. R.: Rispondo continuando il ragionamento svolto nella risposta precedente. Senza una politica fondata su conoscenze dei problemi sociali così come sono esperiti da chi li soffre non si può neanche pretendere di uscire dalla crisi in corso. Come dice tra gli altri un economista quale Bellofiore, nessuna ripresa economica è immaginabile senza una profonda mutazione del tipo di sviluppo ${ }^{32}$. In altri termini, non si può certo pensare di superare la recessione ritornando ad aumentare, ad esempio, la solita produzione di cemento e auto o il solito consumo di telefonini e gadget elettronici. Per dirla in termini marxisti, sono dei nuovi "valori d'uso" che devono essere prodotti e resi disponibili. Ma

\footnotetext{
${ }^{1}$ «Il Gruppo di ricerca di Etnografia del pensiero svolge da anni ricerche in luoghi cruciali della realtà sociale. Presso Aziende, Organizzazioni del Terzo settore, Pubbliche Amministrazioni. Attraverso un'innovativa metodologia di ricerca sociale, raccoglie, qualifica e valorizza idee spesso latenti o trascurate da cui trarre consigli per buone prassi nonché proposte formative per il miglioramento dei luoghi indagati. Il Grep utilizza come risorsa le parole e il pensiero di chi non ha competenze di governo o ricopre ruoli esecutivi. Questo pensiero rappresenta un prezioso capitale intangibile, risorsa decisiva per la gestione di luoghi di lavoro o di servizi».

${ }^{2}$ Si veda anche BELLOFIORE, Riccardo (a cura di), Da Marx a Marx? Un bilancio dei marxismi italiani del Novecento, Roma, Manifestolibri, 2007; ID. (a cura di), Il lavoro di domani. Globalizzazione finanziaria, ristrutturazione del capitale e mutamenti della produzione, Pisa, Biblioteca Franco Serantini, 1998; ID., I lunghi anni Settanta. Crisi sociale e integrazione economica internazionale, in BALDISSARA, Luca, Le radici della crisi. L'Italia tra gli anni Sessanta e gli anni Settanta, Roma, Carocci, 2001, pp. 57-102; ID., La cruna dell'ago. Politica economica e condizioni del lavoro nella crisi del fordismo, in FAVILLI, Paolo, TRONTI, Mario (a cura di), Classe operaia. Le identità: storia e prospettiva, Milano, Franco Angeli, 2001; BALDISSARA, Luca (a cura di), Le radici della crisi. L'Italia tra gli anni Sessanta e gli anni Settanta, Roma, Carocci, 2001.
} 
come si fa a sapere quali, se non chiedendolo, con metodi adeguati d'inchiesta, a chi più patisce della crisi in corso?

L'Italia, tra l'altro gode, di una risorsa ben più ricca di altri paesi Ue: l'afflusso di una recente migrazione quanto mai eterogenea, giovane, prolifica, spesso più qualificata di quel che si riesce a riconoscere. Ma il nostro paese, vecchio e depresso come dicono tutti i dati demografici ed economici -, stenta a utilizzare al meglio tale risorsa. Ciò accade anzitutto perché c'è una forza politica infaustamente decisiva, quale la Lega, che insiste nel tentare di soggiogare i migranti, vessandoli e d umiliandoli, mentre nella sinistra prevale la propensione pedagogica a volerli educare all'“integrazione”. Il tutto mentre anche i rapporti Istat confermano che le stesse tradizioni della società italiana sono in via di crescente disintegrazione.

Ecco allora che se si trattasse davvero di riqualificare la "sinistra" in Italia, un modo ce lo si potrebbe anche immaginare: occorrerebbe spingerla ad occuparsi meno di comunicazione, elezioni e governance, per giovare di più, e con migliore cognizione di causa, all'unità politica di quel "nuovo popolo" che di fatto si sta formando in Italia laddove c'è incontro, e non scontro, amicizia, e non odio, tra popolazioni autoctone e non.

Quale migliore contributo di questo si potrebbe dare a quell'Europa che è tutta da rifare? 


\section{* L'autore}

Valerio Romitelli è ricercatore di Storia delle dottrine politiche nella Facoltà di Scienze politiche dell'Università di Bologna. Studioso di storia della politica italiana ed europea moderna e contemporanea, con particolare attenzione alle questioni metodologiche e d'approccio problematico, è anche ricercatore delle metodologie delle scienze sociali incentrate sulla proposta di un approccio etnografico a distanza da ogni metalinguaggio e volto a conoscere e pensare il pensiero di popolazioni a disagio a partire dalle loro stesse parole. È autore di numerosi libri tra i quali Quando si è fatto politica in Italia? Storia di situazioni pubbliche (1796-1951), Soveria Mannelli, Rubbettino, 2004; Etnografia del pensiero. Ipotesi e ricerche, Roma, Carocci editore, 2005; Fuori della società della conoscenza. Ricerche di etnografia del pensiero, Roma, Infinito Edizioni, 2009.

\section{Per citare questo articolo:}

BUFARALE, Luca, PIETRANCOSTA, Fausto, «Intervista a Valerio Romitelli», Diacronie. Studi di Storia Contemporanea: Quando la classe operaia andava in paradiso, 13/2/2012,

URL:<http://www.studistorici.com/2012/02/13/romitelli_numero_9/ >

\section{Diacronie Studi di Storia Contemporanea $\beta$ www.diacronie.it}

Risorsa digitale indipendente a carattere storiografico. Uscita trimestrale. redazione.diacronie@hotmail.it

Comitato di redazione: Marco Abram - Giampaolo Amodei - Jacopo Bassi - Luca Bufarale - Alessandro Cattunar - Alice De Rensis Barbara Galimberti - Deborah Paci - Fausto Pietrancosta - Martina Sanna - Matteo Tomasoni - Luca Zuccolo

Diritti: gli articoli di Diacronie. Studi di Storia Contemporanea sono pubblicati sotto licenza Creative Commons 2.5 . Possono essere riprodotti a patto di non modificarne i contenuti e di non usarli per fini commerciali. La citazione di estratti è comunque sempre autorizzata, nei limiti previsti dalla legge. 